\title{
Supernova hydrodynamics experiments on the Nova laser*
}

\author{
B. A. Remington, ${ }^{\dagger, a)}$ J. Kane, ${ }^{\text {b) }}$ R. P. Drake, ${ }^{\text {c) }}$ S. G. Glendinning, ${ }^{\text {a) }}$ K. Estabrook, ${ }^{\text {a) }}$ \\ R. London, ${ }^{\text {a) }}$ J. Castor, ${ }^{\text {a) }}$ R. J. Wallace, ${ }^{\text {a) }}$ D. Arnett, ${ }^{\text {b) }}$ E. Liang, ${ }^{\text {d) }}$ R. McCray, $\left.{ }^{e}\right)$ \\ A. Rubenchik, ${ }^{f)}$ and B. Fryxe $\|^{g)}$ \\ a) Lawrence Livermore National Laboratory, Livermore, California 94550 \\ ${ }^{b)}$ University of Arizona, Tucson, Arizona 85721 \\ ${ }^{c)}$ University of Michigan, Ann Arbor, Michigan 48109 \\ ${ }^{d)}$ Rice University, Houston, Texas 77251 \\ ${ }^{e)}$ University of Colorado, Boulder, Colorado 80309 \\ ${ }^{f)}$ University of California, Davis, California 95616 \\ ${ }^{g}$ George Mason University, Fairfax, Virginia 22030
}

(Received 11 November 1996; accepted 8 January 1997)

\begin{abstract}
In studying complex astrophysical phenomena such as supernovae, one does not have the luxury of setting up clean, well-controlled experiments in the universe to test the physics of current models and theories. Consequently, creating a surrogate environment to serve as an experimental astrophysics testbed would be highly beneficial. The existence of highly sophisticated, modern research lasers, developed largely as a result of the world-wide effort in inertial confinement fusion, opens a new potential for creating just such an experimental testbed utilizing well-controlled, well-diagnosed laser-produced plasmas. Two areas of physics critical to an understanding of supernovae are discussed that are amenable to supporting research on large lasers: (1) compressible nonlinear hydrodynamic mixing and (2) radiative shock hydrodynamics. () 1997 American
\end{abstract} Institute of Physics. [S1070-664X(97)91205-2]

\section{INTRODUCTION}

On February 23, 1987 at 0735 UT, the blue supergiant Sanduleak $-69^{\circ} 202$ located in the Large Magellanic Cloud (LMC), a dwarf galaxy at a distance of $50 \mathrm{kpc}$ from earth, exploded as a core-collapse type II supernova (SN). ${ }^{1}$ This event was marked by a prodigious outburst of neutrinos followed $\sim 3 \mathrm{~h}$ later by the UV flash as the shock broke through the surface of the star. Thus began what will certainly be recorded as the most significant astrophysical event of the decade. By February 23, 1997 we will have been studying SN1987A for 10 years; our understanding has progressed enormously. One example is the consensus that strong hydrodynamic mixing of the heavier core elements outwards into the lower density envelope is needed to explain a wide range of observables. This mixing is illustrated in Fig. 1(a) with results from a two-dimensional (2-D) simulation of SN1987A at $3.6 \mathrm{~h}$ after explosion showing an image of density, ${ }^{2}$ and will be discussed further below. But new mysteries continue to emerge. Despite considerable effort world wide, simulations still predict that the mixing front progresses nearly a factor of 2 more slowly than observed. ${ }^{2-8}$

SN1987A is now evolving into the early remnant stage. An optical image taken in Feb. 1994 with a wide-field camera on the Hubble Space Telescope ${ }^{9}$ is shown in Fig. 1(b). The expanding SN ejecta corresponds to the central bright spot, surrounded by what appears to be an assembly of three rings. The origin of these ring nebulae still remains a mystery. ${ }^{10}$ There is general agreement, however, that the SN ejecta is expanding at a much higher velocity $\left(\sim 10^{4} \mathrm{~km} / \mathrm{s}\right)$ than the nebular rings $(10-20 \mathrm{~km} / \mathrm{s})$, with the ejecta expected

*Paper 4IF2, Bull. Am. Phys. Soc. 41, 1475 (1996).

${ }^{\dagger}$ Symposium speaker. to impact the inner ring in 5-10 yr. ${ }^{10-17}$ We stand poised to witness a colliding plasma "astrophysics experiment" of a rather spectacular nature. Simulations offer enticing glimpses of what may transpire, as shown by the density-pressure plots in Figs. 1(c) and 1(d) (reproduced from Ref. 16). Apart from cosmic pyrotechnics, one harbors hope that this collision may shed light on the nature of the circumstellar ring nebula.

Both of these phases of SN evolution [the core hydrodynamic mixing at intermediate times $\left(10^{3}-10^{4} \mathrm{~s}\right)$ and colliding plasmas during remnant formation] are areas rich with possibilities for supporting laboratory experiments. We report here on two such experiments, utilizing the Nova laser ${ }^{18}$ to create the relevant plasma environment. In Sec. II, we discuss hydrodynamic instabilities in the context of corecollapse SN (in particular, SN1987A), and in Sec. III we present the corresponding laser experiment. In Sec. IV, we describe the early stages of SN1987A remnant formation, and the corresponding laser experiment is discussed in Sec. V. Conclusions and an outlook for the future are contained in Sec. VI.

\section{THE HYDRODYNAMICS OF SN1987A}

Supernovae represent one of nature's most dramatic and spectacular exhibitions, with peak luminosities exceeding that of entire galaxies. Observations have not been limited to modern times, either, with such historical examples as Tycho's supernova (SN1572) and Kepler's supernova (SN1604). ${ }^{19}$ Much effort has been invested in developing models to understand the underlying processes of supernovae. Until recently, most efforts have been focused on onedimensional (1-D) stellar evolution models, treating multidimensional hydrodynamics effects with prescriptions from mixing length theory. All this changed with SN1987A. The 
(a)

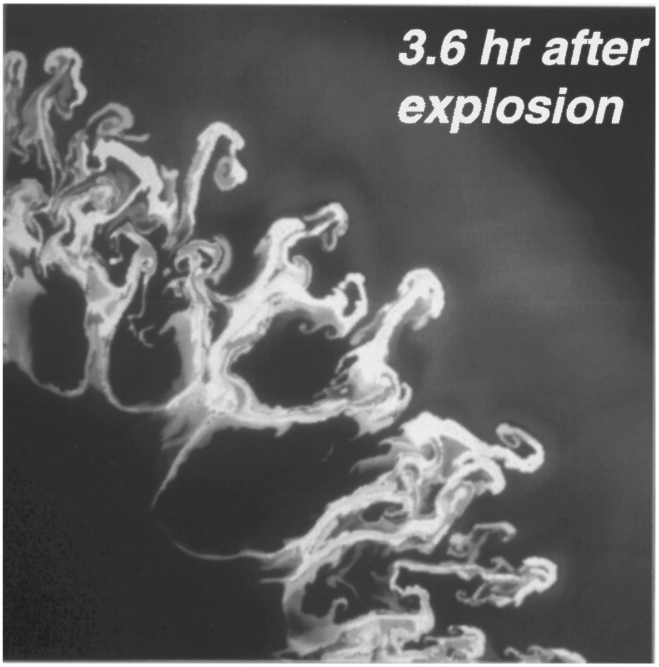

(c)

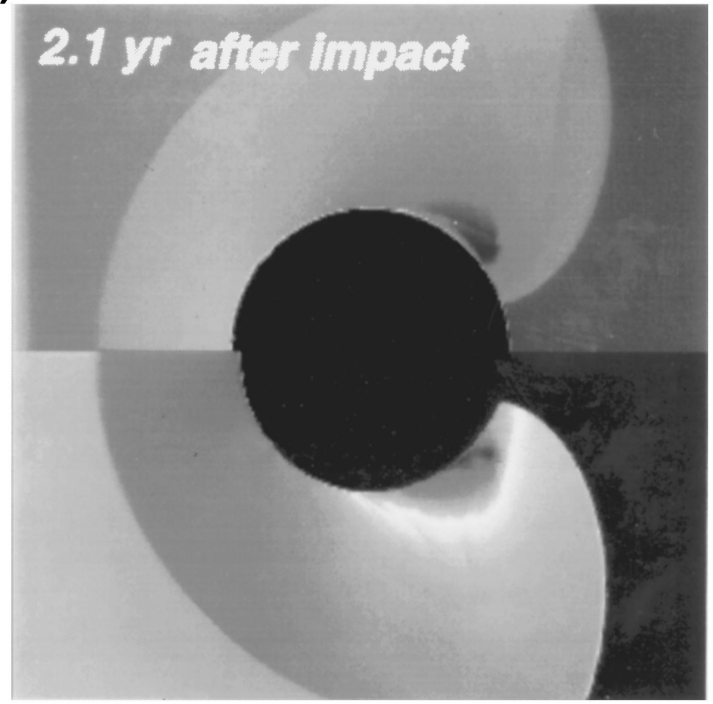

(b)

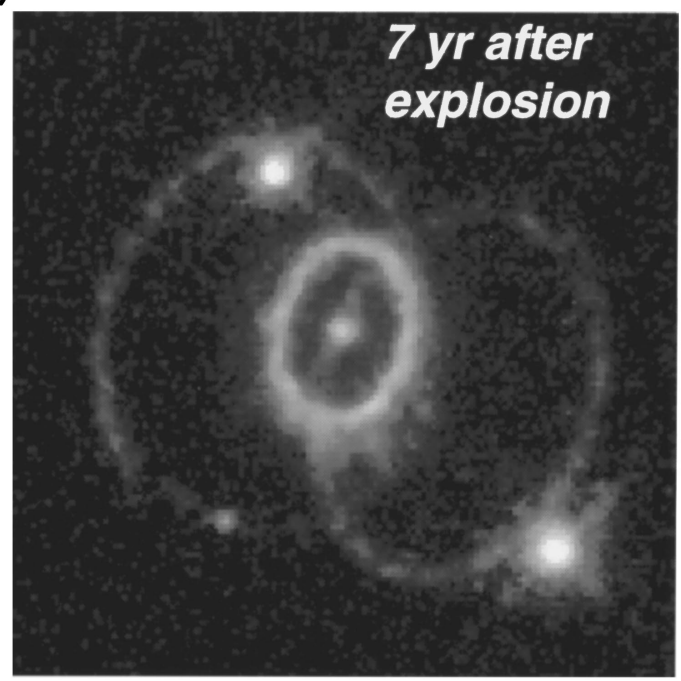

(d)

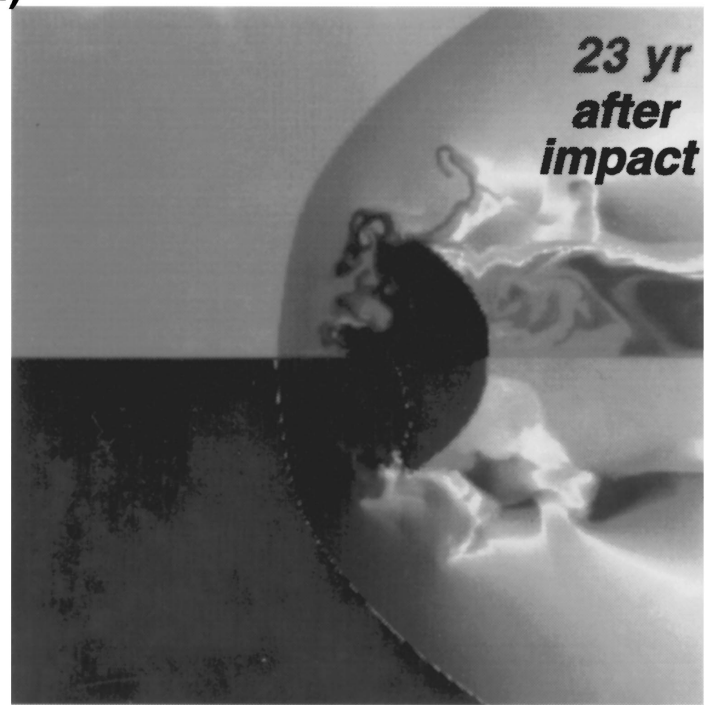

FIG. 1. (a) Density distribution at a time of $3.6 \mathrm{~h}$ after explosion from a 2-D simulation of the hydrodynamic mixing in SN1987A. (Reproduced from Ref. 2.) The spatial extent of the image is $3 \times 10^{12} \mathrm{~cm}$. The seed at the beginning of the calculation for the Rayleigh-Taylor growth was a random perturbation of $10 \%$ amplitude applied to the radial velocity in each zone behind the shock. (b) An image of SN1987A obtained by the Hubble Telescope in Feb. 1994. The expanding supernova ejecta is the central dot. The inner ring is a planetary nebula of uncertain origin. The outer rings are also part of the nebular structure. The emissions have now faded but are expected to resume in a few years when the ejecta strike the inner ring. (Reproduced from Ref. 9.) (c) Simulations of the collision of the SN ejecta with the inner ring nebula two years after initial impact (from Ref. 16). The top half represents density, and the bottom half is pressure. (d) Same as (c) only 23 years after initial impact.

first hints that all was not well with the spherically symmetric 1-D model of SN1987A came from the light curve, that is, total luminosity versus time. A secondary maximum was observed, but considerably earlier ( $\sim 20$ days $)$ and broader than expected. ${ }^{20}$ Then came the "Bochum event," a spectroscopic anomaly starting at day $\sim 25$ suggesting an auxiliary heat source. ${ }^{21}$ The observation of the core elements of ${ }^{56} \mathrm{Ni},{ }^{56} \mathrm{Co}$, and ${ }^{56} \mathrm{Fe}$ poking out through the surrounding hydrogen envelop six months earlier than expected, however, proved conclusive. ${ }^{10,22}$ The 1-D models of SN were largely abandoned, and modeling in 2D commenced in earnest. From the Doppler broadening of the infrared and gamma spectral lines of Fe, Ni, and Co, core velocities of $3000 \mathrm{~km} / \mathrm{s}$ and higher were inferred. ${ }^{10,22}$ Modeling in 2D predicts significantly lower peak velocities ${ }^{2,3}$ of $<2000 \mathrm{~km} / \mathrm{s}$. It would be highly beneficial at this point to provide experimental tests of the codes used to model supernovae.

Current uncertainties notwithstanding, the following picture has emerged for SN1987A. A 1-D stellar evolution calculation gives the density profile for the $19 M_{\odot}$ mass progenitor, ${ }^{23}$ shown in Fig. 2(a). There exists an inner Fe core, $M_{r} / M_{\odot}<1.6$, surrounded by a layer of $\mathrm{Si}, \mathrm{Ne}, \mathrm{O}$, and $\mathrm{C}$ in the region corresponding to $1.6<M_{r} / M_{\odot}<2.3$, followed by a mostly He layer at $2.3<M_{r} / M_{\odot}<6$, and ending in a hydrogen envelope for $M_{r} / M_{\odot}>6$, which extends out to a radius of $R_{0}=2.2 \times 10^{12} \mathrm{~cm}$. Here, $M_{r}$ refers to the mass 

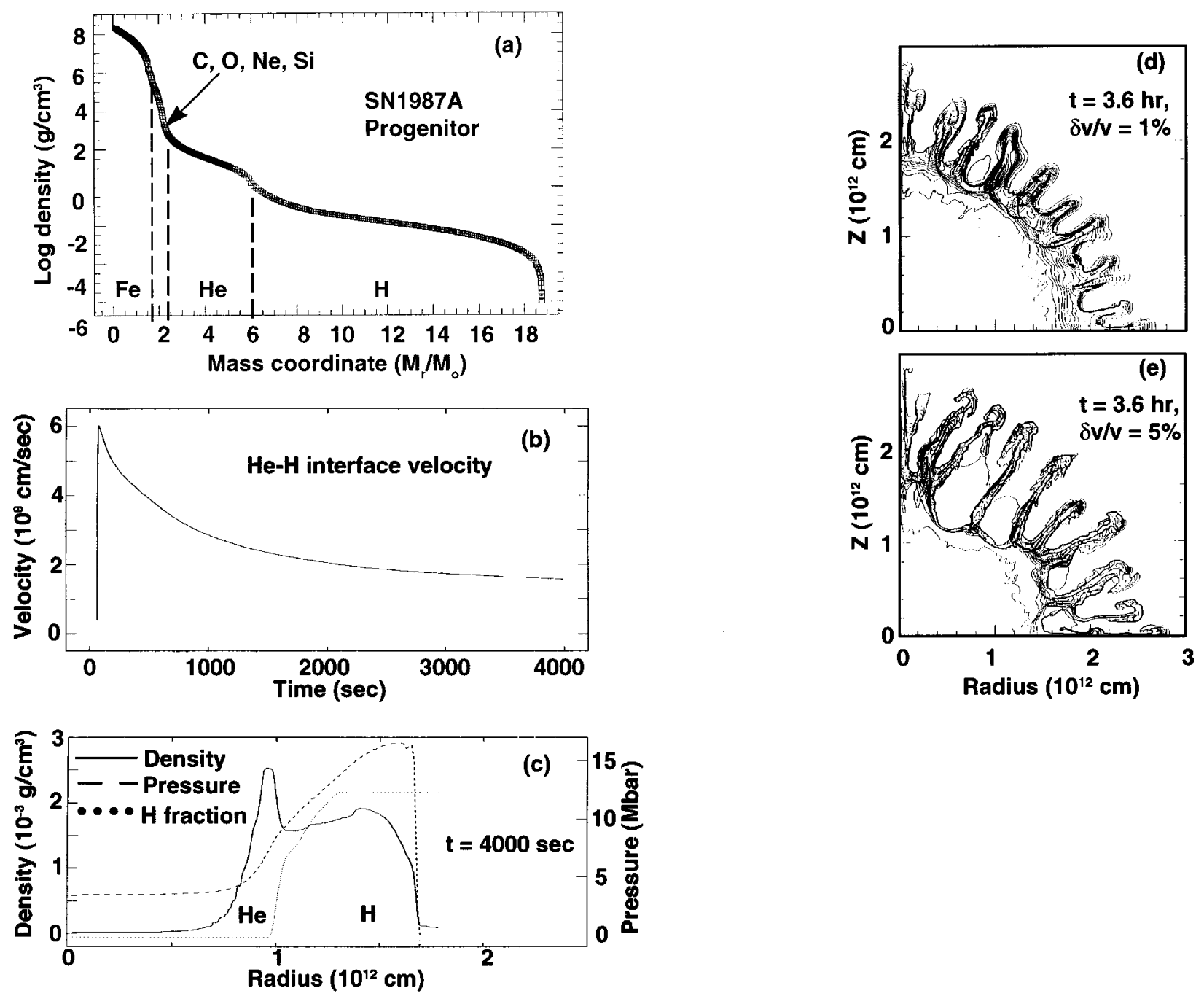

FIG. 2. Supernova simulations in 1D of SN1987A showing (a) the initial density profile $\left(M_{r}\right.$ represents the mass contained out to a radius of $r$, and $M_{\odot}$ is one solar mass), (b) the velocity versus time of the He-H interface, and (c) density, pressure, and $\mathrm{H}$ mass fraction profiles at $4000 \mathrm{~s}$. (d) -(e) Results from 2-D simulations of SN1987A showing isodensity contours of the RT unstable interfaces, corresponding to random multimode seed perturbations in velocity of $\delta v / v=1 \%$ and $5 \%$. (From Ref. 3.)

out to a radius $r$, and $M_{\odot}$ represents one solar mass. The SN explosion is triggered when the Fe core collapses to form a $1.6 M_{\odot}$ neutron star. When the core reaches the density of nuclear degenerate matter, the core rebounds, which launches an exceedingly strong radial shock propagating outward through the star, corresponding to a release of $1-2$ $\times 10^{51}$ ergs of energy, which effectively blows the star apart. The mass cut, that is, the division between what collapses into the neutron star versus what is ejected, is believe to lie somewhere within the oxygen layer.

In the discussion to follow, we start with a progenitor similar to that shown in Fig. 2(a), and calculate the hydrodynamic evolution using the SN hydrodynamics code PROMETHEUS. ${ }^{3,24}$ In this work, we focus on the instabilities at the He-H interface. To economize on computing time, we model only $M_{r} / M_{\odot} \geqslant 5$, depositing the explosion energy, $E=1.5 \times 10^{51} \mathrm{ergs}$, as a mix of thermal and kinetic energy at the inner boundary $\left(M_{r} / M_{\odot}=5\right)$. This launches a strong radial shock that reaches the $\mathrm{He}-\mathrm{H}$ interface $\left(M_{r} / M_{\odot}=6\right)$ after a transit time of about $100 \mathrm{~s}$. At this point, the initial density, pressure, temperature, and velocity at the interface are $2.3 \mathrm{~g} / \mathrm{cm}^{3}, 75 \mathrm{Gbar}, 6 \mathrm{keV}$, and $6 \times 10^{8} \mathrm{~cm} / \mathrm{s}$, respectively. We show the velocity of the $\mathrm{He}-\mathrm{H}$ interface as a function of time in Fig. 2(b), and the density and pressure profiles at a time of $4000 \mathrm{~s}$ in Fig. 2(c). By $4000 \mathrm{~s}$, the shock has traveled about halfway out of the star. Note that at the $\mathrm{He}-\mathrm{H}$ interface $\left(R \approx 1.0 \times 10^{12} \mathrm{~cm}\right)$, the pressure, and density gradients are crossed, that is, $\boldsymbol{\nabla} \rho \cdot \nabla P<0$, such that the He layer is being decelerated by the lower density $\mathrm{H}$ layer. This situation is unstable to the Rayleigh-Taylor (RT) instability ${ }^{25}$ and perturbations at the interface grow in time.

The evolution of compressible, nonlinear, multimode RT instability is an unsolved theoretical problem, and one turns to detailed numerical simulations. An example of a 2-D SN simulation $^{3}$ is shown in Figs. 2(d) and 2(e) by the isodensity contours corresponding to a time of $3.6 \mathrm{~h}$. The two cases shown differ only in the magnitude of the initial multimode root-mean-square (rms) velocity perturbation: $\delta v / v=1 \%$ and $5 \%$. Figure 1 (a) shows a similar calculation only at a factor of 2 higher resolution, ${ }^{2}$ and $\delta v / v=10 \%$. Though 
(a)

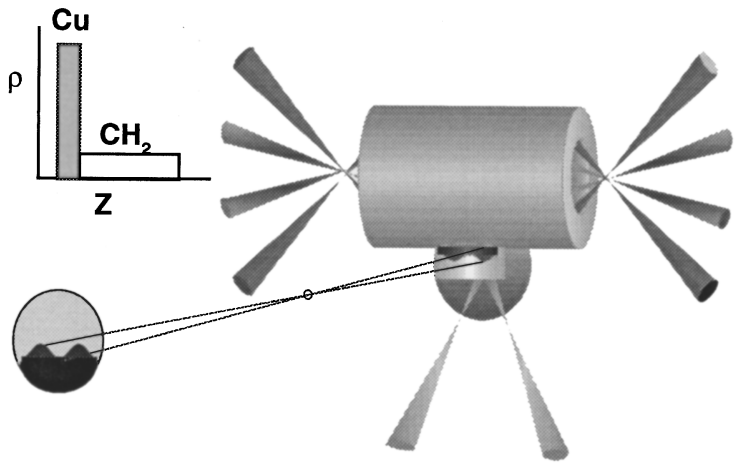

(b)

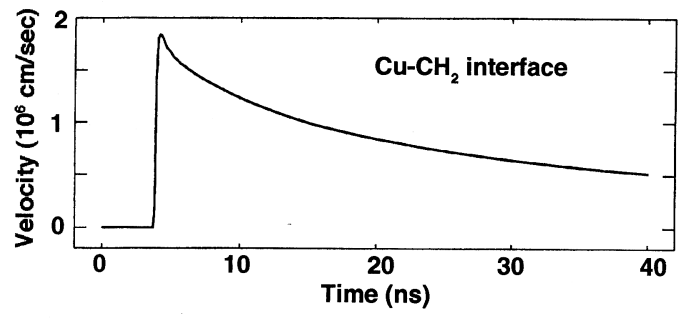

(c) CALE vs HYADES, $t=20 \mathrm{~ns}$

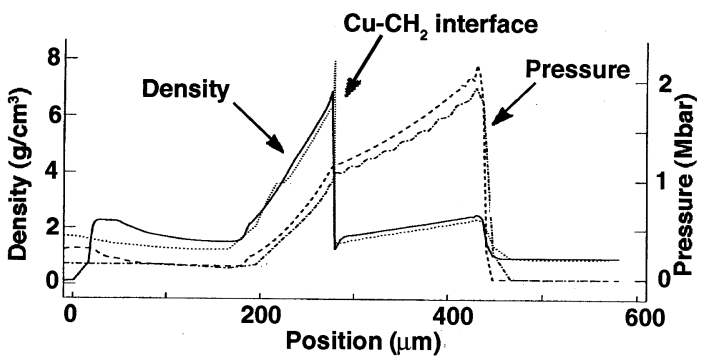

(d) PROMetheus vs HyADES, $t=20$ ns

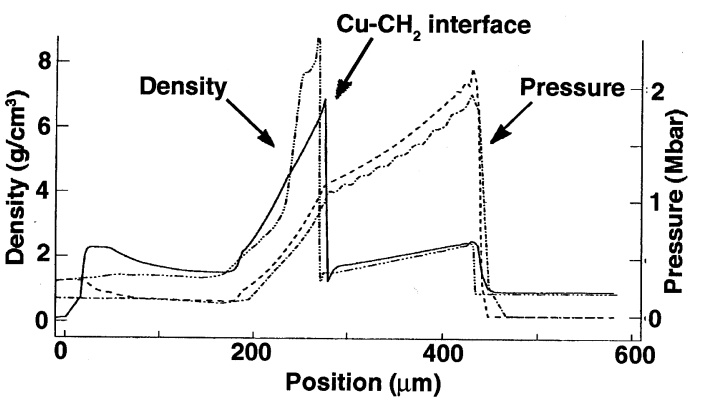

FIG. 3. Supernova hydrodynamics experiment using the Nova laser. (a) Experimental configuration; (b) the velocity versus time of the $\mathrm{Cu}-\mathrm{CH}_{2} \mathrm{interface} \mathrm{from}$ a 1-D HYADES simulation; (c) 1-D simulation showing density and pressure profiles at $20 \mathrm{~ns}$ using the codes HYADES and CALE. The $\mathrm{Cu}-\mathrm{CH}_{2}$ interface is located at a position of about $280 \mu \mathrm{m}$, as indicated, and the forward shock in the $\mathrm{CH}_{2}$ is at about $440 \mu \mathrm{m}$. (d) Same as (c) except using PROMETHEUs instead of CALE.

these seed velocity perturbations are introduced ad hoc here, they are not unreasonable in magnitude, based on recent simulations of convection in the oxygen burning shell just prior to the SN exposion. ${ }^{26}$ There is strong instability growth evident, with spikes of the heavier He falling radially outward through the lower density $\mathrm{H}$ layer. We note the following: (1) The instability has evolved well into the nonlinear regime for all three calculations, with characteristic peak-tovalley amplitudes larger than characteristic wavelengths, $\eta_{\mathrm{PV}} \geqslant \lambda_{\text {char }}$, and the perturbations taking on the classic RT bubble-and-spike shape. (2) The final result at $3.6 \mathrm{~h}$ is still sensitive to the "initial conditions," since increasing the seed amplitudes increases the growth. (3) There appears to be a characteristic dominant mode of mode number $l$ $=2 \pi R / \lambda \approx 20$, though the starting configuration was a random multimode pattern. (4) The peak velocities of the Ni spikes penetrating into the hydrogen envelop in these two calculations are not appreciably different, with $v_{\max }$ $<2000 \mathrm{~km} / \mathrm{s}$.

\section{EXPERIMENTS OF SUPERNOVAE HYDRODYNAMICS}

The experimental configuration adopted for these laser experiments is illustrated in Fig. 3(a) and is described extensively elsewhere. ${ }^{27-29}$ Eight of the ten Nova laser beams at a duration of $1 \mathrm{~ns}$ and total energy of $12 \mathrm{~kJ}$ are focused into a $3.0 \mathrm{~mm}$ long, $1.6 \mathrm{~mm}$ diam Au hohlraum (cylindrical radiation cavity) converting to a $\sim 190 \mathrm{eV}$ thermal x-ray drive. The experimental package is planar, a $85 \mu \mathrm{m} \mathrm{Cu}(\rho$ $\left.=8.9 \mathrm{~g} / \mathrm{cm}^{3}\right)$ foil backed by $500 \mu \mathrm{m}$ of $\mathrm{CH}_{2} \quad(\rho$ $\left.=0.95 \mathrm{~g} / \mathrm{cm}^{3}\right)$. A $\lambda=200 \mu \mathrm{m}$ wavelength, $\eta_{0}=20 \mu \mathrm{m}$ amplitude sinusoidal ripple is imposed at this embedded interface. The package is mounted across a hole in the hohlraum wall, so that the inner (smooth) side of the $\mathrm{Cu}$ sees the $\mathrm{X}$-ray drive. Diagnosis of the interface is through side-on, $\mathrm{x}$-ray radiography, using the remaining two Nova beams focused onto a Fe backlighter disk to generate a 5 ns pulse of $\mathrm{He}-\alpha \mathrm{x}$ rays at $6.7 \mathrm{keV}$. In this side-on view, the opaque $\mathrm{Cu}$ appears as a shadow, and the $\mathrm{CH}_{2}$ is essentially transparent.

We model the laser experiment using a combination of codes. In Figs. 3(b)-3(d) we show the results of modeling in 1-D with HYADES, ${ }^{30}$ CALE, ${ }^{31}$ and PROMETHEUs. ${ }^{3,24}$ HYADES is a 1-D Lagrangian code with multigroup radiation transport and tabular equation of state (EOS), CALE is a 2-D arbitrary Lagrangian-Eulerian code with tabular EOS, and PROMETHEUS is a 3-D Eulerian piecewise parabolic method (PPM) code using (here) an ideal gas EOS. We use a measured radiation temperature, $T_{r}(t)$, as the source input to HYADES. Figure 3(b) shows the velocity of the $\mathrm{Cu}-\mathrm{CH}_{2}$ interface as simulated in HYADES. Note the impulsive shock acceleration, followed by a protracted deceleration, similar to the $\mathrm{He}-\mathrm{H}$ interface in the SN, shown in Fig. 2(b). We do a high-resolution HYADES run, including multigroup radiation transport, for the first $2.45 \mathrm{~ns}$, at which time the shock is approaching the $\mathrm{Cu}-\mathrm{CH}_{2}$ interface. We then map the results to either 1-D or 2-D CALE and PROMETHEUS. (We do not have radiation transport in the versions of these 2-D codes that we are using.) We compare the results for pressure and 

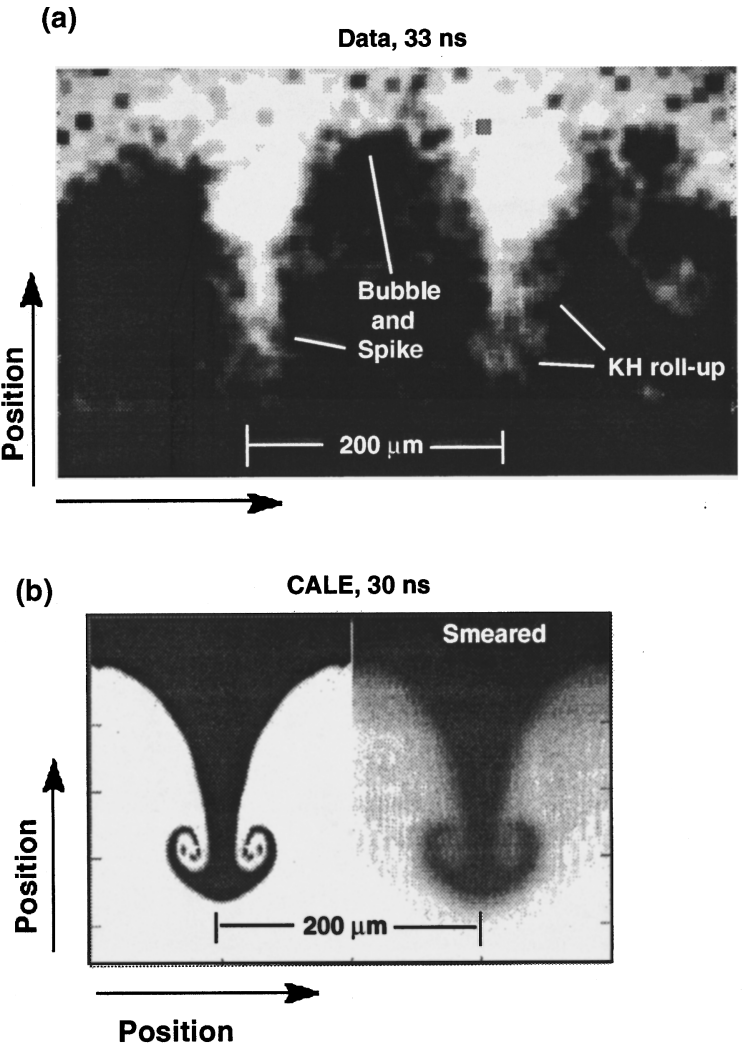

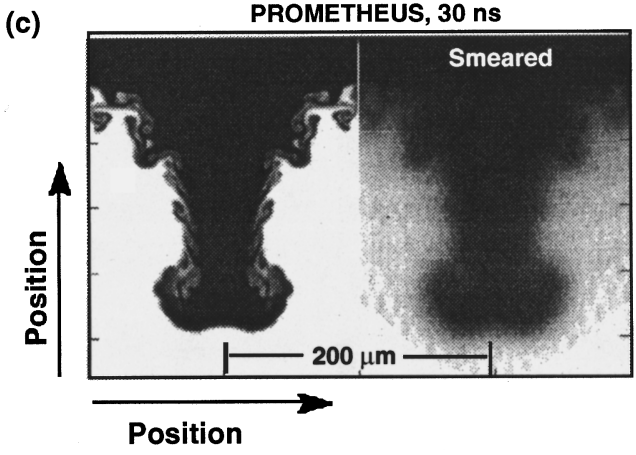

(d)

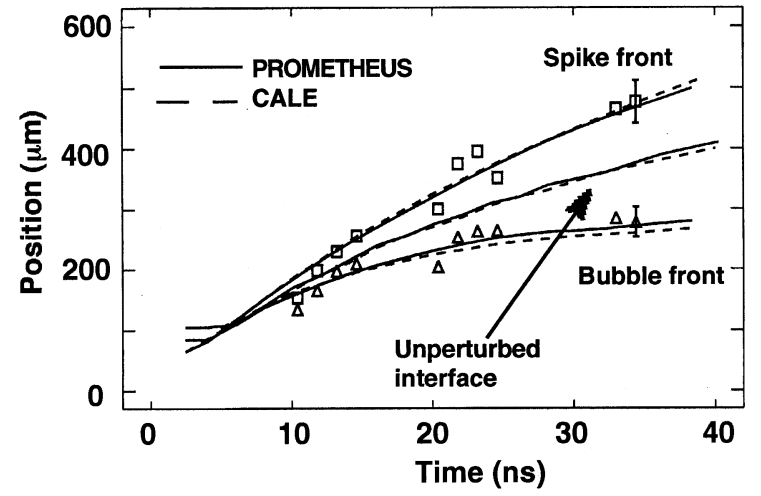

FIG. 4. Comparison of (a) data at 33 ns with the simulations using (b) CALE at $30 \mathrm{~ns}$ and (c) PROMETHEUS. The results labeled "smeared" have been convolved with the experimental instrument resolution function. (d) Comparison of the bubble-and-spike front trajectories observed in the data and from the simulations.

density at $20 \mathrm{~ns}$ from a continuous 1-D HYADES run including radiation transport versus that from CALE [Fig. 3(c)] and PROMETHEUS [Fig. 3(d)]. The mapping works very well for both codes. Note, the pressures for the Nova experiment, 1-2 Mbar, are not too different from those of the SN (10-15 Mbar), as shown in Fig. 2(c), though the SN densities are lower by a factor of about $10^{3}$.

The difference of scales between the SN and the Nova experiment needs to be addressed. If we assume the mixing is dominated by the RT instability, then in the nonlinear regime, the fluid flow can be characterized by a spatial scale of the order of the perturbation wavelength $\lambda$ and velocity of the order of the perturbation terminal bubble velocity $v_{B}$ $\propto(g \lambda)^{1 / 2}$. Here $g$ corresponds to the acceleration and we have assumed a constant Atwood number. A hydrodynamic time scale is then given by $\tau=\lambda / v_{B} \propto(\lambda / g)^{1 / 2}$, and the hydrodynamics equations are invariant under the scale transformation $^{32} \lambda \rightarrow a_{1} \lambda, g \rightarrow a_{2} g$, and $\tau \rightarrow\left(a_{1} / a_{2}\right)^{1 / 2} \tau$. We illustrate this transformation, using characteristic scales taken from the simulations shown in Figs. 2 and 3. At 4000 $\mathrm{s}$ for the $\mathrm{SN}$, the deceleration of the $\mathrm{He}-\mathrm{H}$ interface is $g_{\mathrm{SN}}$ $=-1.5 \times 10^{4} \mathrm{~cm} / \mathrm{s}^{2}$, the density gradient scalelength is $L_{\mathrm{SN}}$ $=\rho / \nabla \rho=8 \times 10^{10} \mathrm{~cm}$, and the dominant perturbation wavelength is approximated to be $\lambda_{\mathrm{SN}} \approx 10 L_{\mathrm{SN}}=8 \times 10^{11} \mathrm{~cm}$. For the Nova experiment at $20 \mathrm{~ns}$, we have $g_{\text {Nova }}=-2.5$ $\times 10^{13} \mathrm{~cm} / \mathrm{s}^{2}, \lambda_{\text {Nova }}=2 \times 10^{-2} \mathrm{~cm}$, and a characteristic time interval of $\tau_{\mathrm{Nova}}=5 \mathrm{~ns}$. The scale transformation is given by $a_{1}=\lambda_{\mathrm{SN}} / \lambda_{\mathrm{Nova}}=4 \times 10^{13}$, and $a_{2}=g_{\mathrm{SN}} / g_{\mathrm{Nova}}=6 \times 10^{-10}$. The corresponding hydrodynamically equivalent time inter- val for the $\mathrm{SN}$ is then given by $\tau_{\mathrm{SN}}=\left(a_{1} / a_{2}\right)^{1 / 2} \tau_{\mathrm{Nova}}=1.3$ $\times 10^{3} \mathrm{~s}$, which is a reasonable time scale for the SN instability evolution that we are investigating. (Similar scale transformations across vastly different scales have been demonstrated experimentally before. ${ }^{33}$ ) This estimate is overly simplistic, in that we have not accounted for decompression, finite layer thickness, and shocks. Nevertheless, the Nova experiment appears to be investigating nonlinear compressible hydrodynamics similar to that at the $\mathrm{He}-\mathrm{H}$ interface of a type II SN at intermediate times $\left(10^{3}-10^{4} \mathrm{~s}\right)$.

In Fig. 4 we show a 2-D image from the experiment at 33 ns [Fig. 4(a)] compared with results from the 2-D simulations at $30 \mathrm{~ns}$ [Figs. 4(b) and 4(c)], both before and after smearing to resemble the effect of the instrumental spatial resolution. The experimentally observed perturbation has evolved into the classic nonlinear RT bubble-and-spike shape with peak-to-valley amplitude $\eta_{\mathrm{PV}} \approx \lambda$, and there are faint indications of Kelvin-Helmholtz roll ups at the tip of the spike and along its sides. For the simulations, we use the same mapping scheme in $2 \mathrm{D}$ as we did in 1-D, only now the $\mathrm{Cu}-\mathrm{CH}_{2}$ interface has a $\lambda=200 \mu \mathrm{m}$ wavelength, $\eta_{0}$ $=20 \mu \mathrm{m}$ amplitude sinusoidal ripple. The run is started at $t$ $=0$, corresponding to when the drive lasers turn on. By 10 ns, a strong ( $\sim 15 \mathrm{Mbar})$ shock has passed through the interface, and the ripple in the $\mathrm{Cu}$ has an inverted phase due to the Richtmyer-Meshkov instability. ${ }^{34}$ By 30 ns the perturbation has grown with the opposite phase to an overall peakto-valley amplitude of $\eta_{\mathrm{PV}} \approx 180 \mu \mathrm{m} \approx \lambda$, as shown in Figs. 
4(b) and 4(c). The shape of the perturbation has changed from sinusoidal to bubble and spike, indicating that the interface has evolved well into the nonlinear regime. Thus, by 30 ns we access roughly the same degree of nonlinearity in the laser experiment as shown for the supernova in Fig. 2(d) for the $1 \%$ velocity perturbation.

The gross features of the experiment are reproduced by both simulations, CALE [shown in Fig. 4(b)] and PROMETHEUS [in Fig. 4(c)]. However, there is more fine structure in the PROMETHEUS simulation. When CALE is run in pure Eulerian mode with ideal gas EOS (not shown), that is, in nearly the same manner as the PROMETHEUS simulation, both codes give similar results. However, there is still somewhat less fine structure in the CALE result due to interface tracking. In Fig. 4(d) we show the evolution of the spikeand-bubble fronts, compared with the predictions from both codes. The locations of the 2-D bubble front and spike front are reproduced very well by both hydrodynamics codes. In the frame of reference of an unperturbed interface (not shown), both the spike and bubble converge to nearly the same constant terminal velocities, $v_{b} \approx v_{s} \approx 3.5-4.0 \mu \mathrm{m} / \mathrm{ns}$. We compare this with the theoretical asymptotic velocities for the 2-D RT instability predicted by Hecht and Alon, ${ }^{32}$ namely, $v_{b, s}=[(1 / 6 \pi) 2 A /(1 \pm A) g \lambda]^{1 / 2}$, where the $+(-)$ in the denominator refer to bubble (spike). For our average conditions of $g \approx 0.35 \mu \mathrm{m} / \mathrm{ns}^{2}, A \approx 0.64$, and $\lambda=200 \mu \mathrm{m}$, the predicted velocities are $v_{b}=1.7 \mu \mathrm{m} / \mathrm{ns}$ and $v_{s}$ $=3.6 \mu \mathrm{m} / \mathrm{ns}$. Our spike velocities agree with the HechtAlon semi-infinite fluid theory, but our bubble velocities are considerably higher. This may be due to the finite thickness of the $\mathrm{Cu}$ layer. The $\mathrm{Cu}$ spike is falling into an essentially infinite reservoir of $\mathrm{CH}_{2}$ plasma, whereas the bubble of $\mathrm{CH}_{2}$ is rising into a thin layer of $\mathrm{Cu}$ (thickness $\approx 60 \mu \mathrm{m}$ $\ll \lambda=200 \mu \mathrm{m}$ ), the result of which would be higher bubble velocities. At the level of nonlinearity accessed here, $\eta_{\mathrm{P} / \mathrm{V}}$ $\approx \lambda, 3$-D effects are not expected to be significant. This is based on theoretical estimates of when a 2-D $\rightarrow 3$-D transition should occur, ${ }^{32}$ and on separate experiments where we would have observed such a transition, had it occurred. ${ }^{35}$

The study of hydrodynamic instabilities in type II supernovae has broader significance than simply checking a detail in a hydrodynamics calculation. Type II SN are used in the expanding photosphere method (EPM) for determining the Hubble constant $\left(H_{0}\right) .{ }^{36}$ This method holds great promise both because SN are bright, allowing a single method to be used to determine distances from 10s of kpc to 100s of Mpc, and because EPM does not rely upon calibration with other secondary distance indicators. The EPM does not correspond to a standard candle, but rather, holds that on a case-by-case basis the light curve (total emitted flux as a function of time) of a type II SN can be calculated absolutely, albeit in 1D. By comparing the observed brightness of the SN with the calculated brightness, one can infer the distance. This technique compares very well with other distance determinations to the LMC, for example, where SN1987 A resides. ${ }^{36}$ Applying the EPM to a number of different $\mathrm{SN}$ at varying distances $(D)$, together with redshift measurements of the recession velocity $\left(\nu_{\text {rec }}\right)$ allows a plot of $\nu_{\text {rec }}$ versus $D$ to be constructed, whose slope is the Hubble constant, $H_{0}$. Note, however, that any RT-induced mixing of the radioactive ${ }^{56} \mathrm{Ni}$ and ${ }^{56} \mathrm{Co}$ core outward into the envelope serves as a heat source, altering the light curve. ${ }^{20}$ Furthermore, any coupling between the mixing front at the $\mathrm{He}-\mathrm{H}$ interface and the photosphere could cause the photosphere to become crenulated. The initial blast wave itself may in fact have a distorted shape due to Vishniac instabilities, ${ }^{37}$ thereby distorting the photosphere from the very beginning. A crenulated or otherwise distorted photosphere could have a larger surface area than predictions from a 1-D spherical calculation. For example, if the photosphere resembled, say, Fig. 2(d), its surface area would be larger and would look statistically similar no matter what angle the SN was viewed from. At the same temperature, $\mathrm{SN}$ with such crenulated photospheres would be brighter than assumed based on 1D spherical calculations. Consider the implications. For a given recession velocity, if the SN were brighter than assumed, they would be further away. This decreases the slope of the $\nu_{\text {rec }}$ versus $D$ plot, reducing $H_{0}$. Since the age of the universe varies inversely as the Hubble constant ${ }^{38,39}\left(\tau_{\text {Univ }} \approx 2 / 3 \times 1 / H_{0}\right)$, the result of crenulated photospheres due to hydrodynamic instabilities would be an older universe. It bears mentioning that a wide variety of techniques have been applied to determine extragalactic distances, ${ }^{39-41}$ with results for $H_{0}$ ranging from as low as $50-55 \mathrm{~km} \mathrm{~s}^{-1} \mathrm{Mpc}^{-1}$ to as high as $85-90$ $\mathrm{km} \mathrm{s}^{-1} \mathrm{Mpc}^{-1}$. The EPM currently leads to a value ${ }^{36}$ of $H_{0}=73 \mathrm{~km} \mathrm{~s}^{-1} \mathrm{MPc}^{-1}$. Experimentally testing any piece of this puzzle, in this case the modeling of the SN hydrodynamic instabilities (and its effect, if any, on EPM), is indeed a worthwhile pursuit.

\section{SUPERNOVAE REMNANT FORMATION}

Supernovae remnant formation is one of the classic problems of astrophysics, leading to such spectacular objects in the sky as the Crab nebula. The basic radiative hydrodynamics underlying SN remnant formation is also of fundamental interest. For example, it is currently thought that the asymptotic result of radiative hydrodynamic instabilities lead to the formation of "hydrodynamic bullets" such as those observed in the Orion molecular cloud. ${ }^{42}$ With SN1987A, we have for the first time the opportunity to watch the timedependent dynamics of the early stages of SN remnant evolution [see Fig. 1(b)]. A schematic of the remnant formation process is given in Fig. 5(a) (taken from Ref. 12). Highvelocity supernova ejecta sweep up the surrounding ambient plasma, left over from the stellar wind of the SN progenitor. At the contact discontinuity (the place where the two plasmas meet), shocks are launched forward into the ambient plasma ("forward shock") and backward into the SN ejecta ("reverse shock"), as illustrated with the 1D density profile shown in Fig. 5(b) (from Ref. 12). Note that when the plasma hydrodynamics includes radiative losses, the compressed ejecta may collapse to a much higher density. The radiation carries heat away lowering the temperature and pressure, thus, making the shocked SN ejecta more compressible. This steepens the density gradient at the contact discontinuity [compare the dashed and solid curves in Fig. 5(b)]. At the contact discontinuity, the pressure and density gradients have opposite signes, that is, $\boldsymbol{\nabla} P \cdot \boldsymbol{\nabla} \rho<0$. Conse- 
(a)

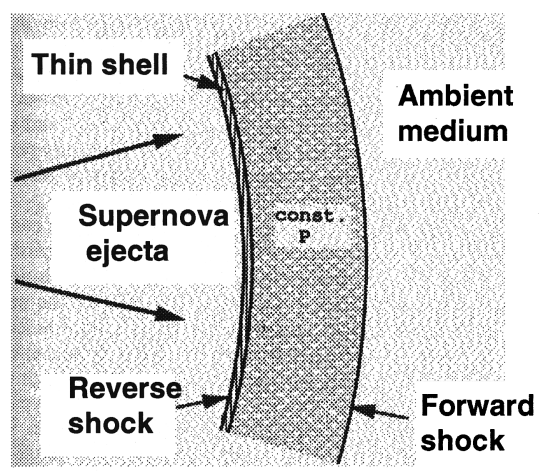

(c)

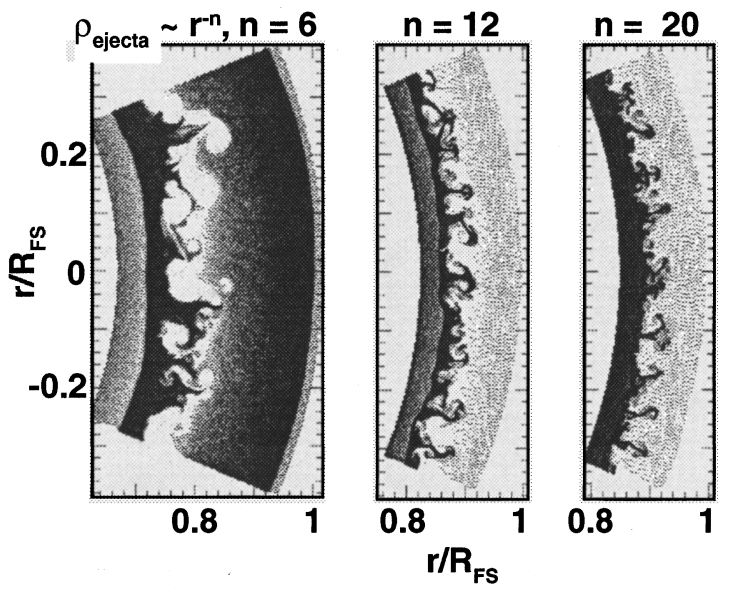

(b)

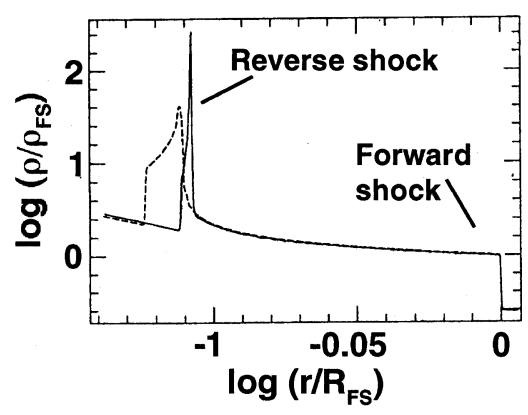

(d)

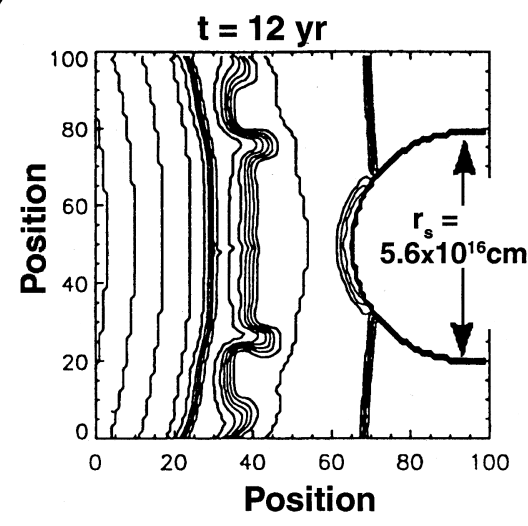

FIG. 5. (a) Schematic showing the dynamics of SN remnant formation. (Reproduced from Ref. 12.) (b) The structure of a generic supernova-stellar wind interaction. The dashed curve shows the structure for negligible radiative power loss. With larger radiative losses, the stagnated ejecta should collapse to higher density, as the solid curve illustrates. (Reproduced from Ref. 12.) (c) 2-D simulations (from Ref. 12) showing the effect of the ejecta density profile, $\rho_{\text {ejecta }} \propto r^{-n}$, on ensuing RT growth at the contact discontinuity. The axes are radius $(r)$ normalized to the position of the forward shock ( $\left.R_{s}\right)$. (d) 2-D simulation result showing RT instability growth at the contact discontinuity, and the imminent collision with the ring. (Reproduced from Ref. 11.)

quently, the shocked circumstellar plasma (of lower density but higher pressure) acts to decelerate the shocked SN ejecta (of higher density but lower pressure). Such a situation is hydrodynamically unstable due to the Rayleigh-Taylor instability. This is illustrated in Fig. 5(c) (from Ref. 12) showing strong RT growth at the contact discontinuity. The simulations assumed a $\rho \propto r^{-n}$ ejecta density profile ( $n$ $=6,12,20$ ) flowing into a uniform ambient plasma. Note that qualitatively different mixing evolves, depending on the density profile of the ejecta. The details of what to expect when the SN1987A ejecta impacts the ring nebula will depend on the structure of both the ring and the projectile assembly. This is further motivated in Fig. 5(d) by the results from a 2-D simulation from a different model (from Ref. 11). Clearly, what transpires will depend upon whether the contact discontinuity looks like Fig. 5(a), 5(c), 5(d), or something completely different. It would be highly beneficial to be able to test these models experimentally prior to the awaited collision.

Hence, our second experiment is focused on testing our understanding of the colliding plasma dynamics in a situation qualitatively similar to that of the ejecta of SN1987A. Our goal is to develop the experiment and model it with the astrophysics codes used to make predictions such as those shown in Figs. 1(c), 1(d), 5(c), and 5(d). This should improve our ability to quantitatively interpret the results of the upcoming pyrotechnics predicted for shortly after the year 2000.

\section{SUPERNOVA REMNANT EXPERIMENT}

Our initial approach to experimentally simulate the ejecta-wind interaction hydrodynamics ${ }^{43}$ is shown in Fig. 6(a). We use about $20 \mathrm{~kJ}$ of laser energy at $0.35 \mu \mathrm{m}$ laser wavelength, in a $1 \mathrm{~ns}$ pulse, to heat a $3 \mathrm{~mm}$ long by $1.6 \mathrm{~mm}$ diameter cylindrical gold cavity (a hohlraum) to a temperature of about $220 \mathrm{eV}$. The x-ray flux ablates a $\mathrm{CH}$ plug, doped with $\mathrm{Br}$ to reduce the transmission of higher-energy $\mathrm{x}$ rays, which is mounted in a $700 \mu \mathrm{m}$ diam hole in the hohlraum. The ablation drives a very strong $(\sim 50 \mathrm{Mbar})$ shock through the $\mathrm{CH}(\mathrm{Br})$, ejecting plasma at about $30 \mathrm{eV}$ from the rear of the plug. This plasma (the ejecta) expands and cools. The leading edge of the expansion is a high-Mach-number plasma flow (about Mach 10), although it is at well below solid density. The ejecta impacts a $700 \mu \mathrm{m}$ diam cylinder of $\mathrm{SiO}_{2}$ aerogel foam located $150 \mu \mathrm{m}$ away and having a den- 

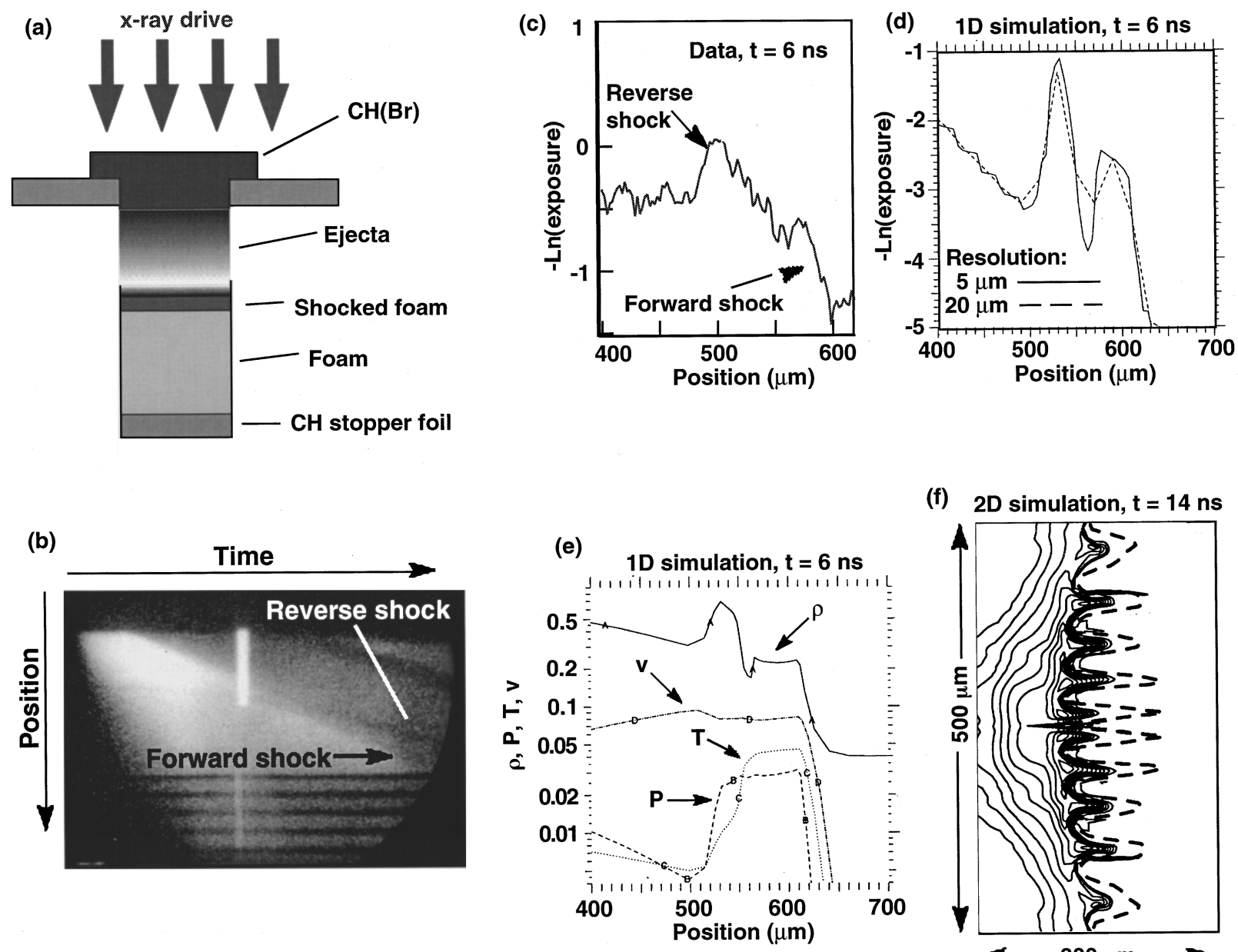

(f) $2 \mathrm{D}$ simulation, $\mathrm{t}=14 \mathrm{~ns}$

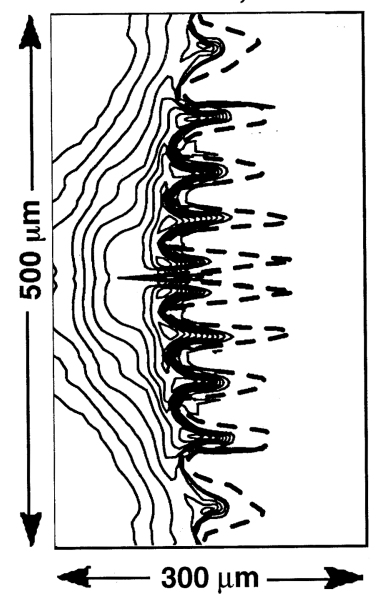

FIG. 6. (a) A schematic of the laser experiment. (b) Raw streaked image from the experiment. (c) A lineout at $6 \mathrm{~ns}$ from the data shown in (b). (d) Same as (c) except based on a 2-D simulation using LASNEX. The solid and dashed lines represent different levels of resolution (5 $\mu \mathrm{m}$ versus $20 \mu \mathrm{m})$. (e) Profiles from the simulation at $6 \mathrm{~ns}$ showing density $\left(\mathrm{g} / \mathrm{cm}^{3}\right)$, pressure $/ 100(\mathrm{Mbar})$, ion temperature $(\mathrm{keV})$, and velocity $\left(\times 10^{8} \mathrm{~cm} / \mathrm{s}\right)$. (f) Isodensity contours at $14 \mathrm{~ns}$ from a 2-D LASNEX simulation, showing perturbation growth due to RT instabilities near the contact discontinuity. The ejecta is flowing into the foam from left to right, and contours in the foam have been suppressed for clarity. The density contours span a maximum of $0.6 \mathrm{~g} / \mathrm{cm}^{3}$ at the base of the RT spikes to $0.2 \mathrm{~g} / \mathrm{cm}^{3}$, in steps of $0.0444 \mathrm{~g} / \mathrm{cm}^{3}$, and the dashed curve represents the contact discontinuity between the ejecta plasma and the ambient foam plasma.

sity of $40 \mathrm{mg} / \mathrm{cm}^{3}$. In response, the flowing ejecta stagnates and a shock is driven into the foam, as well as back into the ejecta.

We diagnose these experiments by $\mathrm{x}$-ray backlighting at $4.3 \mathrm{keV}\left(S c \mathrm{He}_{\alpha}\right)$ to obtain radiographs of the shocked matter. An example is given in Fig. 6(b) showing a 1-D, streaked radiograph image of the target. We show profiles of $-\ln ($ exposure $) \propto$ density from the data in Fig. 6(c) and from a LASNEX ${ }^{44}$ simulation in Fig. 6(d), both at $t=6 \mathrm{~ns}$. In both the data and simulation, we observe a clear forward shock in the foam, a reverse shock in the ejecta, and a contact discontinuity in between. From the simulations, we see that the shock breaks out of the $\mathrm{CH}(\mathrm{Br})$ at about $2 \mathrm{~ns}$, at which time the back edge of the $\mathrm{CH}(\mathrm{Br})$ is at a density of about $2 \mathrm{~g} / \mathrm{cm}^{3}$ (compression of $\sim 2$ ), pressure of $45 \mathrm{Mbar}$, and temperature of $30 \mathrm{eV}$. The foam is impacted by the ejecta about $1 \mathrm{~ns}$ later, suggesting that the high-velocity tail of the ejecta is moving at $\sim 150 \mu \mathrm{m} / \mathrm{ns}=150 \mathrm{~km} / \mathrm{s}$. We show in Fig. 6(e) the density, pressure, temperature, and velocity of the ejecta-foam assembly from the LASNEX simulation at $6 \mathrm{~ns}$, that is, about $3 \mathrm{~ns}$ after the ejecta first starts sweeping up the foam. The contact discontinuity is located at a position of about $560 \mu \mathrm{m}$ in both the data and the simulation, and the peak densities from the simulation on either side of the contact discontinuity in the ejecta (foam) are $0.65 \mathrm{~g} / \mathrm{cm}^{3}$ $\left(0.25 \mathrm{~g} / \mathrm{cm}^{3}\right)$. The pressure is continuous across the contact discontinuity, at a peak value of $3.5 \mathrm{Mbar}$, the peak temperature is about $50 \mathrm{eV}$, and the velocity of the projectile assembly is about $1 \times 10^{7} \mathrm{~cm} / \mathrm{s}$. We point out that both here and in SN1987A, the forward shock driven by the ejecta is a strong shock, that is, the shocked matter is maximally compressed [by a factor of $(\gamma+1) /(\gamma-1)$ for a $\gamma$-law gas].

The region near the contact surface at the front of the ejecta is RT unstable. This is illustrated in Fig. 5(c) for SN1987A, and in Fig. 6(f) for a 2-D simulation of the laser experiment. In the latter, a seed perturbation of wavelength $\lambda=50 \mu \mathrm{m}$ and initial amplitude $\eta_{0}=1 \mu \mathrm{m}$ was imposed on the surface of the foam. By $14 \mathrm{~ns}$, strong RT growth of the perturbation well into the nonlinear regime is visible, due to the $\boldsymbol{\nabla} P \cdot \boldsymbol{\nabla} \rho<0$ configuration at the contact discontinuity (which is indicated by the dashed curve). This bears some semblance to the RT growth shown in Figs. 5(c) and 5(d) for 
the supernova ejecta. We intend to use this experiment to test the theories and models being used to predict the behavior of SN1987A, well in advance of the upcoming SN ejecta-ring nebula impact. This should facilitate the interpretation of the data to emerge from the impact.

\section{CONCLUSIONS AND FUTURE OUTLOOK}

In conclusion, we are developing experiments to investigate (1) hydrodynamic instabilities relevant to corecollapse supernovae at intermediate times $\left(10^{3}-10^{4} \mathrm{~s}\right)$, and (2) plasma flow dynamics relevant to the $\mathrm{SN}$ ejecta-ambient plasma interactions during the early stages of remnant formation. Initial results from both experiments look promising. Expanding the first experiment into 3D is the most critical next step to take, since the RT growth in a supernova is clearly $3 \mathrm{D}$, and growth in $3 \mathrm{D}$ is expected to be larger than in 2D. ${ }^{35,45}$ Extending the second experiment into the radiative regime could also be beneficial, since the remnant formation hydrodynamics of supernovae is often radiative. Experimental results from a French group suggest that a laser-driven radiative hydrodynamics experiment should be possible. ${ }^{46}$

Beginning these astrophysics experiments now on the

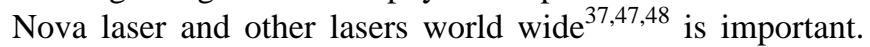
With the construction at Lawrence Livermore National Laboratory in the U.S. of the $\sim 2$ MJ National Ignition Facility laser, ${ }^{49}$ and the similar Laser MegaJoule (LMJ) laser ${ }^{50}$ planned to be built in France, it is crucial that we acquire experience now with developing laser-plasma astrophysics experiments. This ground work will better allow us to plan discriminating astrophysics experiments for the "superlasers" scheduled for completion around the year 2002. Note, this date is just about the time that the SN1987A ejecta is predicted to impact its surrounding nebular ring. Dedication ceremonies for the two superlasers may be consummated with a fitting celestial son et lumière.

\section{ACKNOWLEDGMENTS}

A number of people have been very instrumental in helping this new initiative to get off the drawing boards. The authors are indebted to E. M. Campbell, J. D. Kilkenny, B. A. Hammel, R. Freeman, D. Dearborn, S. Woosley, T. Weaver, P. Pinto, R. Tipton, J. Colvin, and J. T. Larsen for their assistance along the way. The authors also thank R. A. Chevalier for allowing them to reproduce figures from his work in this paper.

This work was performed under the auspices of the U.S. Department of Energy by the Lawrence Livermore National Laboratory under Contract No. W-7405-ENG-48. Partial funding was obtained from LLNL LDRD-ER Grant No. 97ERD-022.

\footnotetext{
${ }^{1}$ W. D. Arnett, J. N. Bahcall, R. P. Kirshner, and S. E. Woosley, Annu. Rev. Astron. Astrophys. 27, 629 (1989); W. Hillebrandt and P. Höflich, Rep. Prog. Phys. 52, 1421 (1989).

${ }^{2}$ E. Müller, B. Fryxell, D. Arnett, Astron. Astrophys. 251, 505 (1991).

${ }^{3}$ B. Fryxell, W. Müller, and D. Arnett, Astrophys. J. 367, 619 (1991).

${ }^{4}$ I. Hachisu, T. Matsuda, K. Nomoto, T. Shigeyama, Astrophys. J. 368, L27 (1991); 390, 230 (1992); Astron. Astrophys. Suppl. 104, 341 (1994).

${ }^{5}$ A. Burrows and B. A. Fryxell, Astrophys. J. 418, L33 (1993).

${ }^{6}$ M. Herant and S. E. Woosley, Astrophys. J. 425, 814 (1994).
}

${ }^{7}$ M. Herant, W. Benz, and S. Colgate, Astrophys. J. 387, 294 (1992); 435, 339 (1994).

${ }^{8}$ H. Bethe, Rev. Mod. Phys. 62, 801 (1990); Astrophys. J. 412, 192 (1993).

${ }^{9}$ A. P. S. Crotts, W. E. Kunkel, and P. J. McCarthy, Astrophys. J. 347, L61 (1989); E. J. Wampler, L. F. Wang, D. Baade, K. Banse, S. D’Odorico, C. Gouiffes, and M. Tarenghi, ibid. 362, L13 (1990); L. Wang and E. J. Wampler, Astron. Astrophys. 262, L9 (1992); P. Jacobsen, R. Albrecht, C. Barbieri, J. C. Blades, A. Boksenberg, P. Crane, J. M. Deharveng, M. J. Disney, T. M. Kamperman, I. R. King, F. Macchetto, C. D. Mackay, F. Paresce, G. Weigelt, D. Baxter, P. Greenfield, R. Jedrzejewski, A. Nota, W. B. Sparks, R. P. Kirshner, and N. Panagia, Astrophys. J. 369, L63 (1991); P. Jakobsen, F. Macchetto, and N. Panagia, ibid. 403, 736 (1993); and on the internet at website /http://www.stsci.edu/pubinfo/jpeg/ SN1987A_Rings.jpg

${ }^{10}$ R. McCray, Annu. Rev. Astron. Astrophys. 31, 175 (1993).

${ }^{11}$ R. McCray and D. N. C. Lin, Nature 369, 378 (1994); D. Luo and R. McCray, Astrophys. J. 379, 659 (1991); 430, 264 (1994).

${ }^{12}$ R. A. Chevalier, J. M. Blondin, and R. T. Emmering, Astrophys. J. 392, 118 (1992); R. A. Chevalier and J. M. Blondin, ibid. 444, 312 (1995).

${ }^{13}$ J. M. Blondin and P. Lundqvist, Astrophys. J. 405, 337 (1993).

${ }^{14}$ C. L. Martin and D. Arnett, Astrophys. J. 447, 378 (1995).

${ }^{15}$ T. Suzuki, T. Shigeyama, and K. Nomoto, Astron. Astrophys. 274, 883 (1993).

${ }^{16}$ K. J. Borkowski, J. M. Blondin, and R. McCray, Astrophys. J. 476, L31 (1997); and "X-rays from the impact of SN 1987A with its circumstellar ring,"' Astrophys. J. (in press).

${ }^{17}$ R. A. Chevalier and V. V. Dwarkadas, Astrophys. J. 452, L45 (1995).

${ }^{18}$ J. D. Kilkenny, Rev. Sci. Instrum. 63, 4688 (1992); E. M. Campbell, Laser Part. Beams 9, 209 (1991).

${ }^{19}$ R. G. Strom, Mon. Not. R. Astron. Soc. 230, 331 (1988).

${ }^{20}$ T. Shigeyama and K. Nomoto, Astrophys. J. 360, 242 (1990).

${ }^{21}$ R. W. Hanuschik and J. Dachs, Astron. Astrophys. 192, L29 (1987); V. M. Blanco, B. Gregory, M. Hamuy, S. R. Heathcote, M. M. Phillips, N. B. Suntzeff, D. M. Terndrup, A. R. Walker, R. E. Williams, M. G. Pastoriza, T. Storchi-Bergmann, and J. Matthews, Astrophys. J. 320, 589 (1987).

${ }^{22}$ F. C. Witteborn, J. D. Bregman, D. H. Wooden, P. A. Pinto, D. M. Rank, S. E. Woosley, and M. Cohen, Astrophys. J. 338, L9 (1989); J. Tueller, S. Barthelmy, N. Gehrels, B. J. Teegarden, M. Leventhal, and C. J. MacCallum, ibid. 351, L41 (1990); R. A. Syunyaev, Nature 330, 227 (1987), Sov. Astron. Lett. 16, 171 (1990); T. Dotani, Nature 330, 230 (1987).

${ }^{23}$ D. Arnett, Supernovae and Nucleosynthesis (Princeton University Press, Princeton, NJ, 1996).

${ }^{24}$ D. Arnett, Astrophys. J. 427, 932 (1994).

${ }^{25}$ Lord Rayleigh, Scientific Papers II, 200, Cambridge, England, 1900; Sir Geoffrey Taylor, Proc. R. Soc. London Ser. A 201, 192 (1950).

${ }^{26}$ G. Bazan and D. Arnett, Astrophys. J. 433, L41 (1994); "Twodimensional hydrodynamics of pre-core collapse: Oxygen shell burning," Astrophys. J. (submitted).

${ }^{27}$ B. A. Remington, S. V. Weber, M. M. Marinak, S. W. Haan, J. D. Kilkenny, R. J. Wallace, and G. Dimonte, Phys. Plasmas 2, 241 (1995).

${ }^{28}$ J. Kane, D. Arnett, B. A. Remington, S. G. Glendinning, J. Castor, R. Wallace, A. Rubenchik, and B. A. Fryxell, "Supernova-relevant hydrodynamic instability experiments on the Nova laser,' Astrophys. J. Lett. (in press).

${ }^{29}$ T. Peyser, P. L. Miller, P. E. Stry, K. S. Bubil, E. W. Burke, D. A. Wojtowicz, D. L. Griswold, B. A. Hammel, and D. W. Phillion, Phys. Rev. Lett. 75, 2332 (1995).

${ }^{30}$ J. T. Larsen and S. M. Lane, J. Quant. Spectros. Radiat. Transf. 51, 179 (1994).

${ }^{31}$ R. Tipton (author of CALE) (private communication).

${ }^{32}$ J. Hecht, U. Alon, and D. Shvarts, Phys. Fluids 6, 4019 (1994); U. Alon, J. Hecht, D. Ofer, and D. Shvarts, Phys. Rev. Lett. 74, 534 (1995).

${ }^{33}$ E. M. Campbell, N. C. Holmes, S. B. Libby, B. A. Remington, and E. Teller, "The evolution of high energy-density physics: From nuclear testing to the superlasers,' Laser Part. Beams (in press).

${ }^{34}$ R. D. Richtmeyer, Commun. Pure Appl. Math. 13, 297 (1960); E. E. Meshkov, Izv. Akad. Nauk SSSR Mekh. Zhidk. Gaza 4, 151 (1969) [Izv. Acad. Sci. USSR Fluid Dynamics 4, 101 (1969)].

${ }^{35}$ M. M. Marinak, B. A. Remington, S. V. Weber, R. E. Tipton, S. W. Haan, K. S. Budil, O. L. Landen, J. D. Kilkenny, and R. Wallace, Phys. Rev. Lett. 75, 3677 (1995); K. S. Budil, B. A. Remington, T. A. Peyser, K. O. Mikaelian, P. L. Miller, N. C. Woolsey, W. M. Wood-Vasey, and A. M. Rubenchik, ibid. 76, 4536 (1996).

${ }^{36}$ B. P. Schmidt, R. P. Kirshner, and R. G. Eastman, Astrophys. J. 395, 366 
(1992); B. P. Schmidt, R. P. Kirshner, R. G. Eastman, M. M. Phillips, N. B. Suntzeff, M. Hamuy, J. Maza, and R. Aviles, ibid. 432, 42 (1994).

${ }^{37}$ J. Grun, J. Stamper, C. Manka, J. Resnick, R. Burris, J. Crawford, and B. Ripin, Phys. Rev. Lett. 66, 2738 (1991); D. Ryu and E. T. Vishniac, Astrophys. J. 313, 820 (1987); 368, 411 (1991); M. M. Mac Low and M. L. Norman, ibid. 407, 207 (1993).

${ }^{38}$ C. J. Hogan, Nature 371, 374 (1994); G. H. Jacoby, ibid. 371, 741 (1994).

${ }^{39}$ D. Branch, E. Baron, P. Nugent, and P. H. Hauschildt, Bull Am. Phys. Soc. 41, 1476 (1996); Phys. Plasmas 4, 2016 (1997).

${ }^{40}$ A. Saha, A. Sandage, L. Labhardt, H. Schwengeler, G. A. Tammann, N. Panagia, and F. D. Macchetto, Astrophys. J. 438, 8 (1995).

${ }^{41}$ G. H. Jacoby, D. Branch, R. Ciardullo, R. L. Davies, W. E. Harris, M. J. Pierce, C. J. Pritchet, J. L. Tonry, and D. L. Welch, Publ. Astron. Soc. Pac. 104, 599 (1992).

${ }^{42}$ J. M. Stone, J. Xu, and L. G. Mundy, Nature 377, 315 (1995); M. M. MacLow, ibid. 377, 287 (1995); D. A. Allen and M. G. Burton, ibid. 363, 54 (1993).

${ }^{43}$ R. P. Drake (private communication).

${ }^{44}$ G. B. Zimmerman and W. L. Kruer, Comments Plasma Phys. Controlled Fusion 2, 51 (1975).

${ }^{45}$ G. Tryggvason and S. O. Unverdi, Phys. Fluids A 2, 656 (1990); J. P.
Dahlburg, J. H. Gardner, G. D. Doolen, and S. W. Haan, Phys. Fluids B 5, 571 (1993); Phys. Plasmas 2, 2453 (1995); J. Hecht, D. Ofer, U. Alon, D. Shvarts, S. A. Orszag, and R. L. McCrory, Laser Part. Beams 13, 423 (1995).

${ }^{46}$ J. P. Le Breton, J. C. Bozier, T. Jalinaud, and J. Valadon, Bull. Am. Phys. Soc. 41, 1479 (1996); J. C. Bozier, G. Thiell, J. P. Le Breton, S. Azra, M. Decroisette, and D. Schirmann, Phys. Rev. Lett. 57, 1304 (1986).

${ }^{47}$ B. H. Ripin, C. K. Manka, T. A. Peyser, E. A. Mclean, J. A. Stamper, A. N. Mostovych, J. Grun, K. Kearney, J. R. Crawford, and J. D. Huba, Laser Part. Beams 8, 183 (1990).

${ }^{48}$ Yu. P. Zakharov, V. M. Antonov, A. V. Melekhov, S. A. Nikitin, A. G. Ponomarenko, V. G. Posukh, V. O. Stoyanovsky, and I. F. Shaikhislamov, in Laser Interaction and Related Plasma Phenomena, 12th International Conference (American Institute of Physics, Woodbury, NY, 1996), p. 357.

${ }^{49}$ J. A. Paisner, E. M. Campbell, and W. J. Hogan, Fusion Technol. 26, 755 (1994).

${ }^{50} \mathrm{M}$. Andre, Revue Scientifique et Technique de la Direction des Applications Militaires (CEA, Paris, 1995), No. 13, M. Andre, First Annual International Conference on Solid State Lasers for Application to ICF, Monterey, CA, 31 May-1 June 1995, SPIE Proceedings (International Society of Optical Engineers, Bellingham, WA, 1996). 